Proceeding of the $1^{\text {st }}$ ICEENG conference, 24-26 March, 1998.

RG. 137

MIIITARY TECHNICAL COLLEGE

CAIRO-EGYPT

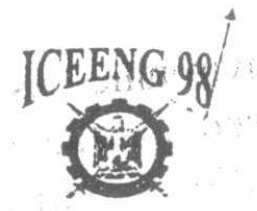

FIRST INTERNATIONAL CONF.ON

ELECTRICAL ENGINEERING

\title{
ASYMPTOTICALLY OPTIMUM DETECTION OF SCINTILLATING TARGETS IMMERSED IN NON-GAUSSIAN INTERFERENCE
}

\author{
Alaa Fahmy" and A. Hanfay"
}

\section{ABSTRACT}

The actual noise environment is often non-Gaussian in nature. In this case, the conventional detector (CD) based on the Gaussian assumption suffers significant performance degradation. To avoid such degradation, an asymptotically optimum detector (AOD) is used. The structure of the AOD is based on an asymptotic expression of the log-likelihood ratio and the functional form of zero memory non linearity (ZMNL), which depends on the noise statistics. In this paper, we calculate the functional form of ZMNL for three different probability density functions (Gaussian, log-normal, and Weibull distribution). The structure of the AOD has been introduced when the probability density function of the noise is either unknown or known. This structure can be used to improve the radar performance.

\section{INTRODUCTION}

In practice, the echo signal from a target in motion is almost never constant. Variations in the echo signal may be caused by meteorological conditions, the lobe structure of the antenna pattern, or equipment instabilities. But the chief source of fluctuation is that of variations in the target cross section. This fluctuation is referred to the target scintillation. That is, the radar cross section vary randomly every time the target is illuminated and may occur between pulses or between scans.

\footnotetext{
"Ph.D., Military Technical College.

** Professor, Military Technical College.
} 
The actual noise environment is often non-Gaussian in nature [1]. Accordingly, conventional detectors based on the Gaussian assumption, when operating in nonGaussian environments, suffer significant performance degradation because of the large tails of the non-Gaussian distributions, which determine higher false alarm rates.

The strategies for detection in non-Gaussian interference, can be categorized into:

1- distribution-free non parametric detection, such as median detectors, rank detectors [2-4];

2- distribution-constrained locally optimum detector (LOD) $[5,6]$ or asymptotically optimum detector (AOD) [7,8]. We concentrate our attention to the AOD category.

\section{DESIGN OF ASYMPTOTICALLY OPTIMUM DETECTOR}

Consider the case where multiple observation are available at the receiver input in the form of signal samples, if a target is present, plus additive noise samples independent of each other and the signal, so that the hypothesis testing problem to be solved is

$$
\begin{aligned}
& \mathbf{H}_{\mathbf{0}}: \mathbf{r}_{\mathbf{i}}=\hat{\mathbf{n}_{\mathrm{i}}} \text {; } \\
& \mathbf{H}_{1}: \mathbf{r}_{\mathbf{i}}=\hat{\mathbf{A}_{\mathbf{i}}} \hat{\mathbf{S}_{\mathbf{i}}}+\hat{\mathbf{n}_{\mathbf{i}}}, \mathbf{i}=1,2, \ldots, \mathbf{N}
\end{aligned}
$$

where,

$r_{i}^{\wedge}=R_{i} \exp \left(-j \theta_{i}\right)$ is the ith sample from the complex envelope of the received signal, $n_{i} \hat{~ i s ~ t h e ~ i t h ~ s a m p l e ~ f r o m ~ t h e ~ c o m p l e x ~ e n v e l o p e ~ o f ~ t h e ~ n o i s e ~(a s s u m i n g ~ t h a t ~ t h e ~}$ variables $\hat{n_{i}}$ are independent and identically distributed I.I.D.), $A_{i} \hat{\mathrm{s}_{\mathrm{i}}} \hat{\text { is }}$ the ith sample from the complex envelope of the useful received signal, modeled as a product of a known shape factor $\hat{s}_{i}=S_{i} \exp \left(-j \varphi_{i}\right)$ times a random amplitude factor $A_{i} \hat{\text {, and }} \hat{s}_{i} \hat{\text { is }}$ the ith sample of the complex envelope of the transmitted signal.

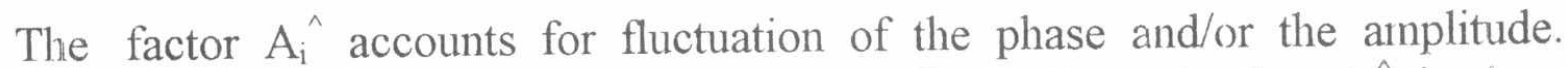
Precisely, if the useful signal is an incoherent non-fluctuating train, then $\mathrm{A}_{\mathrm{i}} \hat{}$ is given by:

$$
\mathrm{A}_{\mathrm{i}} \hat{\wedge}=\mathrm{A} \exp \left(-\mathrm{j} \Psi_{\mathrm{i}}\right)
$$


Proceeding of the $1^{\text {st }}$ ICEENG conference, 24-26 March, 1998.

where, the amplitude $\mathrm{A}$ is a known constant amplitude and the phases $\Psi_{\mathrm{i}}$ are independent random variables uniformly distributed in $(0,2 \pi)$. If the trains fluctuate from scan to scan, then the amplitude $\mathrm{A}$ is a random variable and is expressed as:

$\mathrm{A}=\mathrm{A}_{\mathrm{rms}} \xi$

where, $A_{\text {rms }}$ is the root-mean-square value and $\xi$ is a random variable with unit rootmean-square value representing the normalized amplitude.

If the trains fluctuate from pulse to pulse, then $\mathrm{A}_{\mathrm{i}}{ }^{\wedge}$ will be written as:

$$
A_{i}^{\wedge}=A_{r m s} \xi_{i} \exp \left(-j \Psi_{i}\right)
$$

where, $\xi_{i}, \quad i=1,2, \ldots, N$ are independent identically distributed random variables representing the normalized amplitudes, $\mathrm{A}_{\mathrm{rms}}$ is the common root-mean-square value of the amplitudes. For fluctuating trains, the asymptotically expression of the loglikelihood ratio (LR) is given by:

$$
\gamma_{\mathbf{N}}(\mathbf{R} / \xi)=\sqrt{\varepsilon} \cdot \xi^{2} \cdot \mathbf{I} / \sqrt{\mathbf{N}} \cdot \sum_{\mathbf{i}=1}^{\mathbf{N}} \mathbf{S}_{\mathbf{i}}^{2} \cdot \mathbf{g}\left(\mathbf{R}_{\mathbf{i}}\right)-1 / 2 \cdot \mathbf{I} \cdot \xi^{4} \varepsilon+\alpha_{\mathbf{N}}\left(\mathbf{R}, \mathbf{A}_{\mathrm{rms}}\right)
$$

where, $\varepsilon$ is the energy of the squared envelope of the useful signal, $g(R)$ is the function form of Z'MNL, which depends on the noise statistics and it is given by:

$$
\mathbf{g}(\mathbf{R})=1 / 4 \mathbf{h}(\mathbf{R}) \cdot\left\{\mathbf{d}^{2} \mathbf{h}(\mathbf{R}) / \mathbf{d} \mathbf{R}^{2}+1 / \mathbf{R} \cdot \mathbf{d h}(\mathbf{R}) / \mathbf{d R}\right\}
$$

where, $h(R)$ is the probability density function (PDF) of noise, $I$ is the noise power at the function form of ZMNL and it can be interpreted as an efficiency factor, which is given by:

$$
\mathbf{I}=\mathbf{E}\left\{\mathbf{g}^{2}(\mathbf{R}) / \mathbf{H}_{\mathbf{0}}\right\}
$$

Thus, the asymptotic expression of the LR is given by:

$$
\mathbf{L}_{\mathbf{N}}(\mathbf{R})=\mathbf{E}\left\{\exp \left(\gamma_{\mathbf{N}}(\mathbf{R} / \xi)\right)\right\}
$$

An asymptotically sufficient statistic for all fluctuating models is the variable: 
Proceeding of the $1^{\text {st }}$ ICEENG conference, 24-26 March, 1998.

RG.1 376

$$
\mathbf{D}_{\mathbf{N}}(\mathbf{R})=1 / \sqrt{\mathbf{N}} \cdot \sum_{\mathbf{i}=1}^{\mathbf{N}} \mathbf{S}_{\mathbf{1}}^{2} \cdot \mathbf{g}\left(\mathbf{R}_{\mathbf{i}}\right)
$$

This statistic is compared with the threshold level to decide whether the target is present or not.

\subsection{Structure of A.O.D}

The structure of the A.O.D., as shown in Fig.1, requires processing of the envelope detector by $\mathrm{g}($.) law rather than the quadratic law used in the conventional detector.

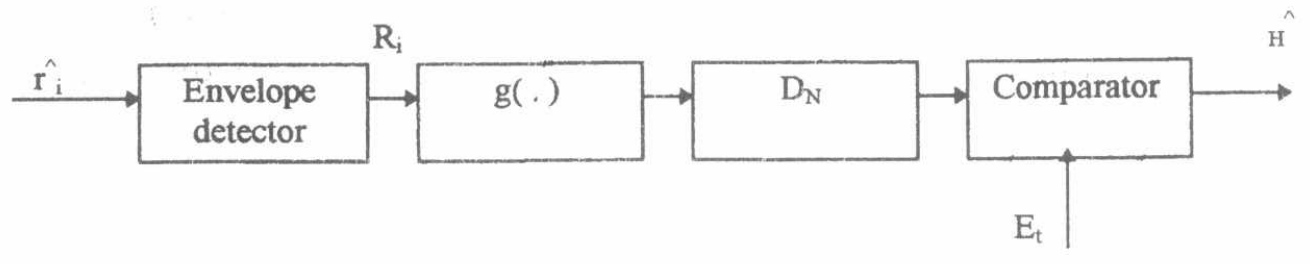

Fig. 1 The structure of A.O.D.

\subsection{Calculation of the Function Form of ZMNL}

In this way, the function form of $\mathrm{ZMNL}$ has been calculated in three cases. The first case, when the marginal PDF of the noise component is assumed to be Gaussian, as given by eq.(10).

$\mathbf{h}(\mathbf{R})=1 / 2 \pi \cdot \sigma^{2} \cdot \exp \left(-\mathbf{R}^{2} / 2 \sigma^{2}\right)$

where, $\sigma^{2}$ is the common variance of the noise quadrature components. Taking the first and second derivative of eq.(10), we get:

$\mathbf{h}^{\prime}(\mathbf{R})=-1 / 2 \pi \cdot \sigma^{2} \cdot \mathbf{R} / \sigma^{2} \cdot \exp \left(-\mathbf{R}^{2} / 2 \sigma^{2}\right)$

$\mathbf{h}^{\prime \prime}(\mathbf{R})=-1 / 2 \pi \cdot \sigma^{4} \cdot \exp \left(-\mathbf{R}^{2} / 2 \sigma^{2}\right)\left(1-\mathbf{R}^{2} / \sigma^{2}\right)$, 
Proceeding of the $1^{\text {st }}$ ICEENG conference, 24-26 March, 1998.

RG.1 377

respectively. Substituting by eqs.(10,11\&12) into eq.(6), we get the function form of ZMNL:

$$
\mathbf{g}(\mathbf{R})=1 / 2 \sigma^{2}\left(\mathbf{R}^{2} / 2 \sigma^{2}-1\right)
$$

It is obvious that $\mathrm{g}(\mathrm{R})$ is equivalent to the conventional square law, but the only difference is the presence of a bias term, which is required in the AOD approach.

The second case, when the marginal PDF of the noise component is assumed to be log-normal distribution, as given by eq.(14) [9]:

$$
\mathbf{h}(\mathbf{R})=\exp \left\{-\ln \left(\sqrt{\mathbf{m}} \cdot \mathbf{R} / \mathbf{R}_{\mathbf{r m s}}\right) / \ln (\mathbf{m})\right\} / 2 \pi \sqrt{\pi \ln (\mathbf{m})} \cdot \mathbf{R}^{2}
$$

where $R_{r m s}$ is the r.m.s envelope, and $m$ is the mean to median radar cross section. Taking the first and second derivative of eq.(14), we get:

$\mathbf{h}^{\prime}(\mathbf{R})=-\Omega \mathbf{R}^{2} / 2 \mu-1 / \chi \cdot \mathbf{R}^{3} . \Psi$,

$\mathbf{h}^{\prime \prime}(\mathbf{R})=\Omega \Psi-(1-3 \mu) /\left(2 \mu^{2} / \Omega \mathbf{R}^{2}\right)+\Omega / \mu \mathbf{R}+3 /\left(\pi \chi \mathbf{R}^{4}\right) . \Psi$,

respectively. Substituting by eqs.(14,15 \&16) into eq.(6), we get the function form of ZMNL:

$$
\mathbf{g}(\mathbf{R})=1 / \mathbf{R}^{2}\left\{(1+\mu)^{2} / \ln (\mathbf{m})-1 /(2 \ln (\mathbf{m}))\right\}
$$

where,

$$
\begin{aligned}
& \mu=\ln (\sqrt{\mathbf{m}}) \cdot \mathbf{R} / \mathbf{R}_{\mathbf{r m s}} ; \\
& \chi=\sqrt{\pi \cdot \ln (\mathbf{m})} ; \\
& \Psi=\exp \left(-\mu^{2} / \ln (\mathbf{m})\right) ; \\
& \Omega=\left(2 \mu^{2} / \pi \chi \ln (\mathbf{m}) \mathbf{R}^{3}\right) . \Psi ; \\
& 2 \mu^{2} \Psi / \Omega \mathbf{R}^{2}=\pi \chi \ln (\mathbf{m}) \cdot \mathbf{R} .
\end{aligned}
$$

The third case, when the PDF of the noise is assumed to be Weibull distribution as given by [4]: 
where $\alpha>0, \beta>0$, and $\mathrm{R}>0$. Taking the first and second derivative, we get:

$\mathbf{h}^{\prime}(\mathbf{R})=-\alpha \beta^{1-2 \alpha} \cdot \mathbf{R}^{2(\alpha-1)} \cdot \phi+(\alpha-1) \cdot \mathbf{R}^{\alpha-2} \cdot \phi$,

$\mathbf{h}^{\prime \prime}(\mathbf{R})=-\alpha^{2} \beta^{1-3 \alpha} \cdot \mathbf{R}^{3 \alpha-3} \cdot \phi-2 \alpha(\alpha-1) \beta^{1-3 \alpha} \cdot \mathbf{R}^{2 \alpha-3} \cdot \phi+\alpha(\alpha-1) \beta^{-\alpha} \cdot \mathbf{R}^{2 \alpha-3} \cdot \phi+$ $(\alpha-1)(\alpha-2) \cdot \mathbf{R}^{\alpha-3} \cdot \phi$,

respectively. Where, $\phi=\exp \left(-\mathrm{R}^{\alpha} \beta^{-\alpha}\right)$. Substituting by eqs.(18,19 \&20) into eq.(6), we get the function form of $\mathrm{ZMNL}$ :

$$
\begin{aligned}
& \mathbf{g}(\mathbf{R})=1 / 4 \mathbf{R}^{2}\left\{\alpha(\alpha-2) \beta^{\alpha-1}+\alpha(\alpha-1) \mathbf{R}^{\alpha} \beta^{-1}-2 \alpha(\alpha-1) \mathbf{R}^{\alpha} \beta^{-2 \alpha}+\right. \\
& \left.\alpha^{2} \mathbf{R}^{2} \beta^{-2 \alpha}-\alpha \mathbf{R}^{\alpha} \beta^{-\alpha}+\beta^{\alpha-1}\right\} .
\end{aligned}
$$

Let $\alpha=2, \beta=2$, therefore,

$$
\mathbf{g}(\mathbf{R})=1 / 16 \mathbf{R}^{2}\left(\mathbf{R}^{4}+\mathbf{R}^{2}+4\right) .
$$

Moreover if the PDF of the noise is unknown/ known, therefore, the structure of the AOD is shown in Fig. 2 and Fig. 3 respectively. In Fig.2, the envelope detector output is sampled and these sampled data is stored in a memory unit, the joint PDF of the noise is calculated, and then the marginal PDF of the noise. The function form of ZMNL $g(R)$ is calculated by special purpose computer. Finally the statistic $D_{N}$ is calculated and compared with the threshold level $E_{t}$, to decide whether the target is present or not.

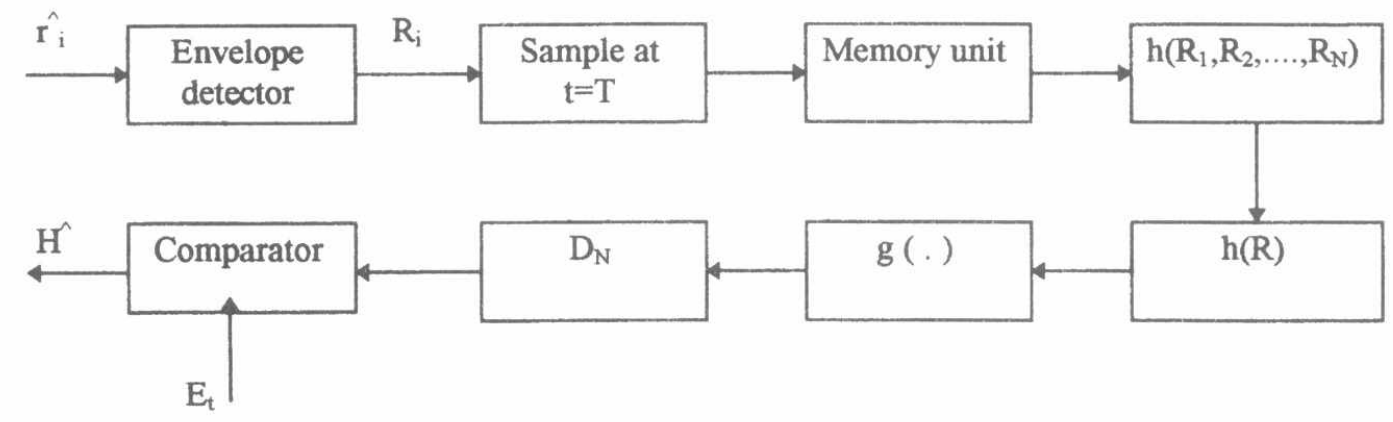

Fig.2 Structure of AOD with unknown PDF of noise 
Proceeding of the $1^{\text {st }}$ ICEENG conference, 24-26 March, 1998.

In Fig. 3, the envelope detector output is sampled and these sampled data is applied to analog to digital converter $(\mathrm{ADC})$. The output from the $\mathrm{ADC}$ is then applied to a special purpose computer, which calculates the function form of $Z M N L L(R)$. Hence, the function form of ZMNL is applied to digital to analog converter (DAC). Finally the statistic $D_{N}$ is calculated and compared with the threshold level $E_{t}$, to decide whether the target is present or not.

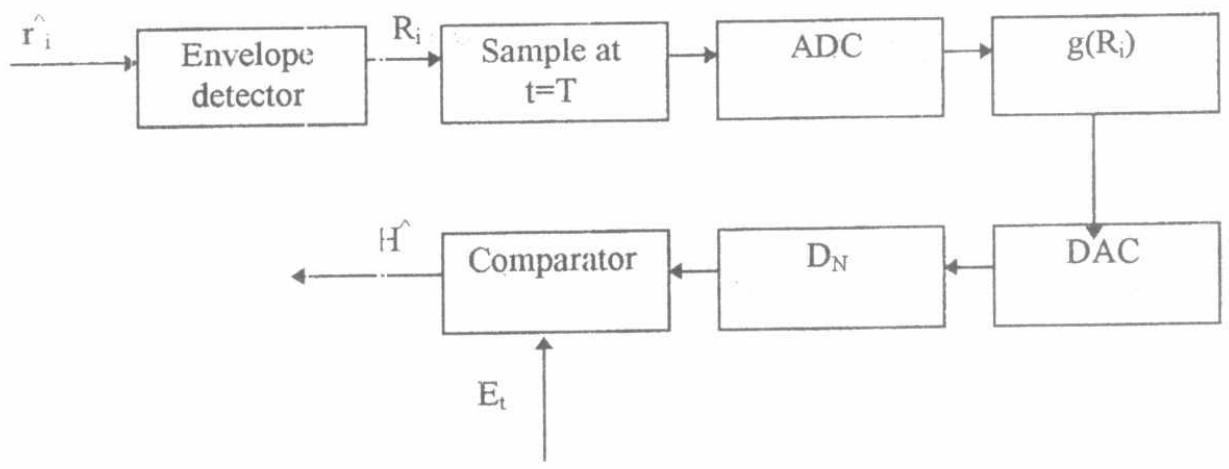

Fig.3 Structure of AOD with known PDF of noise

\section{ASYMPTOTIC PERFORMANCE}

The asymptotic statistic of the decision variable $D_{N}$ is given by:

$$
\begin{aligned}
& \mathbf{H}_{\mathbf{o}}: \mathbf{D}_{\mathbf{N}} \rightarrow \mathbf{N}(0, \mathbf{I}), \\
& \mathbf{H}_{1}: \mathbf{D}_{\mathbf{N}} \rightarrow \mathbf{N}(\mathbf{I} \sqrt{\varepsilon}, \mathbf{I}),
\end{aligned}
$$

where $\mathrm{N}$ denotes the normal distribution. Therefore, the asymptotically operating characteristics in terms of both the probability of false alarm Pfa and the probability of detection $\mathrm{Pd}$ are giver by:

$$
\begin{aligned}
& \mathbf{P}_{\mathrm{fa}}=\mathbf{Q}\left(\mathbf{E}_{\mathbf{t}}\right), \\
& \mathbf{P}_{\mathbf{d}}=1-\mathbf{Q}\left(\sqrt{\varepsilon} \cdot \mathbf{I}-\mathbf{E}_{\mathfrak{t}}\right)
\end{aligned}
$$

where, $Q\left(E_{t}\right)$ is the probability that a standard Gaussian variant exceeds the threshold $E_{t}$. Define the signal to noise ratio as: 
Proceeding of the $1^{\text {st }}$ ICEENG conference, 24-26 March, 1998.

RG. 380

$\Re=\mathbf{A}_{\text {rms }}^{2} / \sigma^{2}$,

therefore, the energy when $\mathrm{N}$ diverges can be expressed as:

$\sqrt{\varepsilon}=\Re \cdot \sigma^{2} \sqrt{\mathbf{N}}$

The probability of detection $\mathrm{Pd}$ in terms of the signal to noise ratio is given by:

$\mathbf{P}_{\mathbf{d}}=1-\mathbf{Q}\left(\mathfrak{R} \sqrt{\mathbf{N} \cdot \mathbf{I}_{\mathbf{n}}}-\mathbf{E}_{\mathbf{t}}\right)$

where, $\mathrm{I} n$ is the normalized improvement factor given by:

$$
\mathbf{I}_{\mathbf{n}}=\mathbf{I} \cdot \boldsymbol{\sigma}^{4}
$$

The efficiency factor given by eq.(7), can be calculated for the ZMNL of eq.(17),

$$
\mathbf{I}=\mathbf{m}^{6}\left\{1+2 \ln \left(\mathbf{m}^{-1}\right)+0.5 \ln \left(\mathbf{m}^{-2}\right)\right\} / \mathbf{R}_{\mathbf{r m s}} .
$$

Therefore, we simulate the calculation of the probability of detection of scintillating targets for different probabilities of false alarm. Figure 4 shows a comparison between the probability of detection for scintillating targets, by using the AOD, immersed in non-Gaussian and that immersed in white Gaussian noise. It is found that the former is better than the latter by about $1.1 \%$.

\section{CONCLUSION}

The calculation of the function form of zero memory non linearity for three different probability density functions, namely, Gaussian, log-normal, and Weibull distribution, has been introduced. Moreover, It has been introduced a structure of the asymptotic optimum detector for unknown and known probability density functions. This structure can be generalized to improve the detection process of the western radar station, when the targets are immersed in non-Gaussian interference. 
Proceeding of the $1^{\text {st }}$ ICEENG conference, 24-26 March, 1998.

\section{REFE'RENCE'S}

[1] Schleher, D.C., "Clutter models and data in automatic detection and radar data processing", Artech house, 1980, P37-61.

[2] Trunk, G.V., "Non-Rayleigh sea clutter: properties and detection of targets", NRL report 7986, 1976.

[3] Schleher ,D.C., "Radar detection in log-normal clutter", Proceedings of IEEE international radar conference, Arlington, VA, 21-23 April, 1975, P262-267.

[4] Schleher, D.C., "Radar detection in Weibull clutter", IEEE Trans., 1976, AES-12, P736-743.

[5] Spaulding, A.D. and Middleton, D., "Optimum reception in an impulsive interference environment", Pt. 1 : coherent detection; Pt. 2: incoherent reception, ibid., 1977, com-25, P910-934.

[6] Modestino, J.W., and Ningo, A.Y., "Detection of weak signals in narrow band non-Gaussian noise", ibid., 1979, IT-25,P592-600.

[7] Fedele, G., Izzo, L. and Pura, L., "Optimum and sub-optimum space diversity detection of weak signals in non-Gaussian noise", IEEE Trans. comm.9, 1984, P990997.

[8] Conte, E., Izzo, L., Longo, M. and Paura, L., "Asymptotically optimum radar detection in sea clutter", Proc. of Mediterranean electro-technical conference, Madrid, 8-10 Oct., 1985, Vol.II: digital signal processing, P339-343.

[9] Conte, E. and Longo, M.,"Asymptotically optimum detection of fluctuating targets in non-Gaussian interference", IEE Proc. Vol.134, Oct. 1987. 


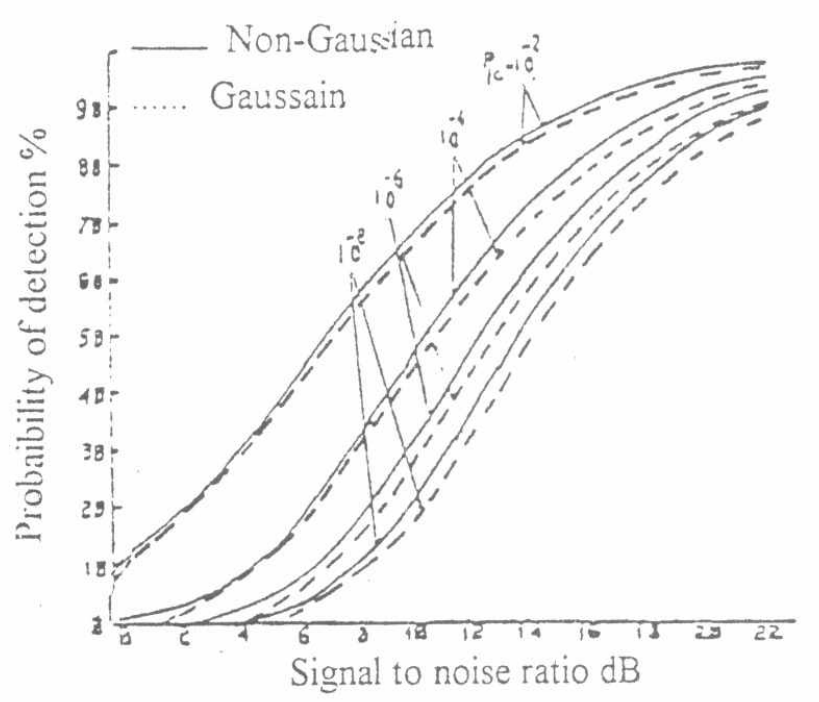

Fig. 4 Probability of detection vs signal to noise ratio for scintillating targets 